\title{
High-performance pentacene thin-film transistor with ZrLaO gate dielectric passivated by fluorine incorporation
}

Chuan Yu Han ${ }^{a}$, Ling Xuan Qian ${ }^{a}$, Cheung Hoi Leung ${ }^{a}$, Chi Ming Che ${ }^{b}$, Pui To Lai ${ }^{a}$, Senior Member, IEEE

${ }^{a}$ Department of Electrical and Electronic, the University of Hong Kong, Hong Kong

${ }^{b}$ Department of Chemistry, the University of Hong Kong, Hong Kong

Corresponding author: P. T. Lai

e-mail: laip@eee.hku.hk

Postal address: Room 505, CYC Building, Pokfulam Road, the University of Hong Kong, Hong Kong

Phone number: 852-28592691 


\begin{abstract}
Pentacene thin-film transistor with high- $\kappa \mathrm{ZrLaO}$ gate dielectric has been fabricated for the first time. After treating the dielectric in a fluorine plasma, the carrier mobility of the transistor can be greatly improved to $0.717 \mathrm{~cm} / \mathrm{V} \cdot \mathrm{s}$, which is more than 40 times that of one without plasma treatment. The major reasons should be larger pentacene grains and fewer traps in the device with gate dielectric passivated by the fluorine plasma. AFM confirms that relatively large and high pentacene islands form on the plasma-treated dielectrics in the initial growth stage, and the growth pattern obviously follows the Vollmer-Weber growth model. Furthermore, the surfaces of the dielectrics with different plasma treatment times are investigated by AFM, XPS and contact-angle measurement to reveal the mechanism / effects of the fluorine incorporation. Lastly, after exposure to atmosphere without encapsulation for 6 months, all the devices still display good transistor characteristics.
\end{abstract}

Keywords Organic thin-film transistor, high- $\kappa$ dielectric, $\mathrm{ZrLaO}$, fluorination 


\section{Introduction}

Organic thin-film transistors (OTFTs) have attracted considerable research interest due to their commercial opportunities, such as flexible display, large-area sensor and radio-frequency identification tags [1-3]. The operation and performance of an OTFT are highly dependent on the interface between its gate dielectric and organic material. To improve the carrier mobility of the OTFT, much attention has been paid to interface modification, including self-assembled monolayer [4], annealing [5] and plasma treatment. Among them, plasma treatment is efficient and convenient to improve the surface characteristics in surface engineering. Oxygen plasma [6, 7], $\mathrm{N}_{2} \mathrm{O}$ plasma [8] and fluorine plasma [9] have been reported to modify the dielectric surface and good results were obtained. Moreover, fluorine-based plasma has long been investigated to provide valuable surface properties in organic and inorganic material fields [10-14]. Generally, the operating voltage of OTFTs based on $\mathrm{SiO}_{2}$ gate dielectric is very high. Avoiding this drawback by reducing the thickness of the gate dielectric inevitably leads to large gate leakage current. Therefore, high- $\kappa$ dielectric used as the insulator layer is a better alternative to lower the threshold voltage, and also thin the device to make it more flexible [15].

In this study, high- $\kappa \mathrm{ZrLaO}$ is proposed as the gate dielectric for pentacene OTFT, and is treated in fluorine plasma for surface modification. With appropriate plasma treatment time, the OTFT can achieve high performance, e.g. high carrier mobility as well as small threshold voltage. Moreover, after 6-month exposure to atmosphere, the devices can still show good transistor characteristics.

\section{Experimental details}

Pentacene OTFTs were fabricated by employing the bottom-gate top-contact configuration. Heavily-doped silicon wafers (n-type, $<100>$, resistivity of $0.5 \sim 0.7 \Omega \cdot \mathrm{cm}$ ) were cleaned according to the standard RCA method and dipped in dilute HF acid $(2 \%)$ to remove the native oxide. Next, $\mathrm{ZrLaO}$ insulator layer was sputtered at room temperature by radio-frequency sputterer (Denton Vacuum LLC Discovery 635). Then, the dielectric was annealed at $400{ }^{\circ} \mathrm{C}_{\text {in }} \mathrm{NH}_{3}$, at a flow rate of $1000 \mathrm{~mL} / \mathrm{min}$ for 10 minutes. After that, the samples were divided into four groups, which were treated in a fluorine plasma, at a flow rate of 10 sccm for $\mathrm{CHF}_{3}$ and $1 \mathrm{sccm}$ for $\mathrm{O}_{2}$, for different durations of $0 \mathrm{~s}, 100 \mathrm{~s}, 300 \mathrm{~s}$ and $600 \mathrm{~s}$. Then, 30-nm or 3-nm pentacene (p-type organic semiconductor, 99\% purity, purchased from Sigma-Aldrich without purification) was evaporated on the dielectrics by an evaporator (Edwards Auto 306). The films were evaporated in high vacuum $\left(5 \times 10^{-6}\right.$ torr) on the dielectrics at a deposition rate of $1.2 \mathrm{~nm} / \mathrm{min}$, monitored by a quartz-crystal oscillator. Lastly, drain and source electrodes were deposited on the 30-nm pentacene films by gold evaporation through a shadow mask to form the OTFTs. The width and length of the channel on the shadow mask were $200 \mu \mathrm{m}$ and $30 \mu \mathrm{m}$, respectively. On the other hand, the 3-nm (about 2 monolayers) pentacene films were used to study their growth mechanism in the initial stage.

The I-V characteristics of the transistors were measured by an HP 4145B semiconductor parameter analyzer. The measurements were repeated after the samples were stored in atmosphere without encapsulation for 6 months. Al/ZrLaO/heavily-doped $\mathrm{Si}$ structure was used to fabricate capacitors by lithography for measuring the dielectric capacitance, which was measured by an HP 4284A precision LCR meter. The thickness of the dielectric films was measured by a Wvase 32 ellipsometer. A Nanopics 2100 atomic force profilometer was employed to record the surface morphology of the dielectrics and the growth of the first few pentacene layers to reveal the mechanism / effects of the fluorine plasma treatment. All the measurements were conducted at room temperature, in air, and under environment shielded from light and electromagnetic wave.

\section{Results and discussions}

\subsection{OTFT characterization}

Typical output characteristics of the pentacene OTFTs plasma-treated for different times are shown in Fig. 1. It indicates that these OTFTs have good field-effect properties and saturation behavior, even after 6 months. All the transistors can operate with a low supply voltage of less than $5 \mathrm{~V}$. Among them, the OTFT with 600-s plasma treatment achieves the largest drive current of above $5 \mu \mathrm{A}$ (fresh) and $2 \mu \mathrm{A}$ (after 6 months), while the OTFT without plasma treatment has the smallest drive current of about 0.6 $\mu \mathrm{A}$ (fresh) and $0.2 \mu \mathrm{A}$ (after 6 months).

The transfer characteristics of the devices are shown in Fig. 2, from which the field-effect carrier mobility can be extracted by the MOSFET model (shown to be readily applicable to OTFTs by Horowitz [16]). In the saturation regime:

$$
I_{D}=(W / 2 L) C_{o x} \mu\left(V_{G}-V_{T}\right)^{2}
$$

where $W$ is the channel width, $L$ the channel length, $C_{o x}$ the insulator capacitance per unit area, $I_{D}$ the saturation current, $V_{G}$ the gate voltage and $V_{T}$ the threshold voltage.

From equation (1), the field-effect carrier mobility can be calculated as

$$
\mu=\frac{2 L\left(\frac{\partial I_{D}{ }^{1 / 2}}{\partial V_{G}}\right)^{2}}{W C_{o x}}
$$


The sub-threshold swing is defined as

$$
S S=\frac{d V_{G}}{d\left(\log I_{D}\right)}
$$

Assuming that both the density of deep bulk trap and density of (deep) interface trap are independent of energy, the sub-threshold swing can be written as [17]

$$
S S=\frac{k T \ln 10}{e}\left[1+\frac{e}{C_{o x}} N_{\mathrm{t}}\right]
$$

where $N_{t}$ is the density of deep bulk trap and the interface trap per unit area and unit energy, $k$ the Boltzmann's constant, and $T$ the temperature in Kelvin. Therefore, the trap density can be extracted from SS by using equation (4).

From the electrical characteristics, the key parameters of the devices can be extracted, and are summarized in Table 1 and 2 . The carrier mobility increases with the plasma treatment time, and the highest carrier mobility is achieved for the 600-s plasma-treated sample, reaching a high value of $0.717 \mathrm{~cm}^{2} / \mathrm{V} \cdot \mathrm{s}$, which is more than 40 times that for the sample without plasma treatment. The on/off current ratio also improves with increasing plasma treatment time. The threshold voltages of the devices are all very small because high- $\kappa \mathrm{ZrLaO}$ is employed as their gate dielectric. In addition, after exposing to atmosphere for 6 months, the OTFTs still show good electrical properties as shown in Fig. 1b, Fig. 2b and Table 2, despite having lower carrier mobility due to degraded pentacene film caused by the moisture and oxygen in air. This should be attributed to the stable interface between pentacene and $\mathrm{ZrLaO}$ because the trap density of the devices hardly changes after 6 months. Lastly, many papers reported that hysteresis in the current-voltage characteristics can be caused by the traps in the pentacene and also in the dielectric, and thus widely exists in organic transistors. Since the fluorine plasma treatment can passivate the traps at the dielectric surface, it should be able to reduce the hysteresis.

\subsection{Pentacene island characterization}

The carrier mobility is a key parameter to characterize the OTFT, and generally is controlled by the grain size of the organic film $[18,19]$, surface scattering caused by rough dielectric surface [20] and dangling bond [21]. Pentacene film growth on the dielectric in the initial stage is crucial to the carrier mobility of organic device because charge transport in the device is mainly confined to the first few monolayers of the organic film in close proximity to the gate dielectric [22]. In order to study the difference in the initial pentacene growth on the $\mathrm{ZrLaO}$ dielectrics, AFM images of 3-nm (about 2 monolayers) pentacene thin films are shown in Fig.3. Even though only 3-nm (about 2 monolayers) pentacene was deposited, the height of the pentacene islands can reach about $10 \mathrm{~nm}$ for the samples with 0 -s and 100-s plasma treatment; about $20 \mathrm{~nm}$ for the dielectrics with 300 -s and 600 -s plasma treatment. The surface morphology is analyzed by a grain analysis tool, and the grain parameters are listed in Table 3 . With quite high pentacene islands and the ratio of grain area far less than $100 \%$, it is clear that the pentacene growth pattern is typically in 3-D clusters according to the Vollmer-Weber growth model $[23,24]$. Also, the pentacene growth seems to form quite high pentacene islands on the dielectrics before one layer is fully formed. The pentacene islands become larger and higher for longer plasma treatment time up to $300 \mathrm{~s}$. Beyond $300 \mathrm{~s}$, it seems that the size of pentacene islands reaches a maximum and then slightly decreases with increasing plasma treatment time. The ratio of grain area also decreases a little.

\subsection{Dielectric properties}

With increasing fluorine-plasma treatment time, more fluorine is incorporated into the $\mathrm{ZrLaO}$ dielectric and thus fewer traps remain at the surface of the dielectric (see Table 1 and Table 4) due to the formation of bonding between fluorine atom and dangling bond. After a 600-s treatment, the atomic concentration of $\mathrm{F}$ in the dielectric can reach $20.3 \%$. In Fig. 4(a), the La $3 d$ spectrum of the untreated sample shows doublet distinct peaks located at $834.7 \mathrm{eV}\left(\mathrm{La} 3 d_{5 / 2}\right)$ and $851.7 \mathrm{eV}\left(\mathrm{La} 3 d_{3 / 2}\right)$, corresponding to $\mathrm{La}-\mathrm{O}$ bonding in the $\mathrm{La}_{2} \mathrm{O}_{3}$ film [25]. After the fluorine plasma treatment, the $\mathrm{La} 3 d$ spectrum is obviously shifted to a higher binding energy by $\sim 1.6 \mathrm{eV}$, indicating that $\mathrm{F}$ is bonded with $\mathrm{La}$ due to its strong electronegativity. Similar phenomenon also appears in the Zr $3 d$ spectrum shown in Fig 4(b), demonstrating the formation of stronger Zr-F bonding. Furthermore, the XPS spectrum of $\mathrm{F} 1 s$ for the plasma-treated samples also confirms the formation of La-F (F 1s 684.5eV) and Zr-F (F 1s 685.1eV) bonds (from the NIST Database) as shown in Fig.5 (a)-(c). Moreover, Fig.5 (d) shows the leakage behavior of ZrLaO dielectrics with different plasma treatment times. The leakage current decreases as the fluorine plasma treatment time increases due to trap passivation by the fluorine plasma.

The surface energies of the samples listed in Table 4, are evaluated using a KRUSS DAS 30 goniometer by the method proposed by Owens and Wendt (Equation (5))[26] based on measuring the contact angles of three liquids, water, diiodomethane and glycerol, at $20^{\circ} \mathrm{C}$ in static mode.: 


$$
1+\cos (\theta)=\frac{2\left(\gamma_{S}^{d}\right)^{1 / 2}\left(\gamma_{L}^{d}\right)^{1 / 2}}{\gamma_{L}}+\frac{2\left(\gamma_{S}^{p}\right)^{1 / 2}\left(\gamma_{L}^{p}\right)^{1 / 2}}{\gamma_{L}} .
$$

where $\gamma_{S}^{d}$ is the dispersion and $\gamma_{S}^{p}$ the polar components of surface energy. The total surface energy $\gamma_{S}$ is obtained by summing the dispersion and polar components. The surface energy, dispersion component and polar component values used to solve Equation (5) are, respectively, 77.2, 22.0, and $50.2 \mathrm{mJm}^{-2}$ for water, and 50.8, 48.5, and 2.3 mJm $\mathrm{Jm}^{-2}$ fiiodomethane, and 64 , 34 , and $30 \mathrm{mJm}^{-2}$ for glycerol. The total surface energy of the $\mathrm{ZrLaO}$ dielectric increases rapidly to $57.8 \mathrm{mJm}^{-2}$ within $100 \mathrm{~s}$, and then slowly to $62.2 \mathrm{mJm}^{-2}$. The size of pentacene island increases with the surface energy due to the fact that a surface with higher surface energy repels the nonpolar pentacene molecules, which can then attract each other more easily, thus resulting in larger pentacene islands [27].

The surface roughness of the dielectric is considered to be one important parameter to decide the pentacene growth, and reduction in grain size and carrier mobility with increasing roughness were reported [20, 28]. However, in this work, the fluorine plasma treatment on the $\mathrm{ZrLaO}$ dielectric results in rougher surface, but the rougher surface does not lead to lower carrier mobility. In the first $300 \mathrm{~s}$, both pentacene-island size and carrier mobility increase as illustrated in Fig. 3 and listed in Table 1, respectively. This implies that the fluorine plasma treatment leads to the increase of the pentacene-island size, which has a stronger effect on the carrier mobility than the roughness increase. The reason is that the fluorine plasma can passivate the dielectric surface to reduce its defects, which act as nucleation sites for pentacene growth. Fewer nucleation sites also result in larger pentacene-island [29]. After $300 \mathrm{~s}$, it can be seen that the pentacene islands on the 300-s and 600-s plasma-treated dielectrics have nearly the same size. However, the latter has much higher carrier mobility than the former. The carrier scatterings caused by rough dielectric surface and traps on the dielectric should govern the carrier mobility when grain sizes are similar. The 600-s plasma-treated surface is a little smoother than the 300-s one as shown in Fig.6. Moreover, the surface with 600-s plasma treatment is passivated for longer time, and thus has much less traps for scattering the carriers. This can be further supported by the smaller value of subthreshold slope (SS) and larger value of on/off current ratio of the corresponding OTFT in Table 1 because both parameters are highly related to the trap density at the dielectric/pentacene interface [30]. Therefore, the carrier mobility of the 600-s plasma-treated sample is much higher than that of its $300-$ s counterpart.

Finally, we can conclude that within the first $300 \mathrm{~s}$ of the plasma treatment, large pentacene islands caused by the fluorine plasma play a key role in carrier-mobility enhancement. However, beyond $300 \mathrm{~s}$, the pentacene islands cannot grow larger, and thus the surface roughness and traps begin to govern the carrier transport.

\section{Conclusion}

We have investigated the effects of fluorine plasma treatment on pentacene thin-film transistor with $\mathrm{ZrLaO}$ proposed as gate dielectric. With plasma-treated dielectric, the properties of the OTFT, especially the carrier mobility, are greatly improved. The results suggest that fluorinated $\mathrm{ZrLaO}$ is a promising gate dielectric for high-performance pentacene TFTs. Moreover, the growth pattern of the pentacene film on the fluorinated $\mathrm{ZrLaO}$ is revealed to follow the Vollmer-Weber growth model. For the first $300 \mathrm{~s}$ of the plasma treatment, the passivated surface can result in larger pentacene islands and thus higher carrier mobility. Beyond 300 $\mathrm{s}$, the size of the pentacene islands remains basically unchanged, and so surface roughness and traps play the key role in the conduction mechanism.

\section{Acknowledgements}

The authors would like to thank Dickey Ma and Linfeng Deng for their help and assistance. This work is supported by the URC for Seed Fund for Strategic Research Theme of HKU on Molecular Materials, and the University Development Fund (Nanotechnology Research Institute, 00600009) of the University of Hong Kong. 


\section{References}

[1] H. Klauk, Organic thin-film transistors, Chem. Soc. Rev., 39 (2010) 2643-2666.

[2] C.D. Dimitrakopoulos, P.R.L. Malenfant, Organic thin film transistors for large area electronics, Adv. Mater., 14 (2002) 99-117.

[3] T. Someya, H.E. Katz, A. Gelperin, A.J. Lovinger, A. Dodabalapur, Vapor sensing with $\alpha, \omega$-dihexylquarterthiophene field-effect transistors: The role of grain boundaries, Appl. Phys. Lett., 81 (2002) 3079.

[4] S.A. DiBenedetto, A. Facchetti, M.A. Ratner, T.J. Marks, Molecular Self-Assembled Monolayers and Multilayers for Organic and Unconventional Inorganic Thin-Film Transistor Applications, Adv. Mater., 21 (2009) 1407-1433.

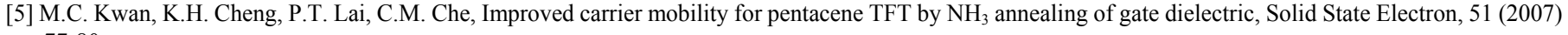
$77-80$.

[6] M.W. Lee, C.K. Song, Oxygen plasma effects on performance of pentacene thin film transistor, Jpn. J. Appl. Phys. Part 1-Regular Papers Short Notes \& Review Papers, 42 (2003) 4218-4221.

[7] K. Shin, S.Y. Yang, C. Yang, H. Jeon, C.E. Park, Oxygen plasma treatment and postaging of pentacene field-effect transistors for improved mobility, Appl. Phys. Lett., 91 (2007) 023508.

[8] C.L. Fan, T.H. Yang, C.C. Lin, C.H. Huang, $\mathrm{N}_{2} \mathrm{O}$-plasma effect on low-temperature deposited gate dielectric for organic thin-film transistors, Electron. Lett., 44 (2008) 1158-1159.

[9] L.F. Deng, Y.R. Liu, H.W. Choi, C.M. Che, P.T. Lai, Improved Performance of Pentacene OTFTs With HfLaO Gate Dielectric by Using Fluorination and Nitridation, Ieee T Device Mat Re, 12 (2012) 520-528.

[10] S. Huang, H. Chen, K.J. Chen, Effects of the fluorine plasma treatment on the surface potential and Schottky barrier height of $\mathrm{Al}_{\mathrm{x}} \mathrm{Ga} \mathrm{1}_{-\mathrm{x}} \mathrm{N} / \mathrm{GaN}$ heterostructures, Appl. Phys. Lett., 96 (2010) 233510.

[11] W.J. Ha, S. Chhajed, S.J. Oh, S. Hwang, J.K. Kim, J.H. Lee, K.S. Kim, Analysis of the reverse leakage current in AlGaN/GaN Schottky barrier diodes treated with fluorine plasma, Appl. Phys. Lett., 100 (2012) 132104.

[12] C.X. Wang, T. Suga, Investigation of fluorine containing plasma activation for room-temperature bonding of Si-based materials, Microelectron. Reliab., 52 (2012) 347-351.

[13] S. Kirk, M. Strobel, C.Y. Lee, S.J. Pachuta, M. Prokosch, H. Lechuga, M.E. Jones, C.S. Lyons, S. Degner, Y. Yang, M.J. Kushner, Fluorine Plasma Treatments of Polypropylene Films, 1-Surface -Characterization, Plasma Process Polym., 7 (2010) 107-122.

[14] R.M. Chu, C.S. Suh, M.H. Wong, N. Fichtenbaum, D. Brown, L. McCarthy, S. Keller, F. Wu, J.S. Speck, U.K. Mishra, Impact of CF 4 plasma treatment on GaN, IEEE Electr Device L, 28 (2007) 781-783.

[15] M.F. Chang, P.T. Lee, S. P.McAlister, S. Albert Chin, Small-Subthreshold-Swing and Low-Voltage Flexible Organic Thin-Film Transistors Which Use HfLaO as the Gate Dielectric, IEEE Electr Device L, 30 (2009) 133-135.

[16] G. Horowitz, Organic Field-Effect Transistors, Adv. Mater., 10 (1998) 365-377.

[17] W.L. Kalb, B. Batlogg, Calculating the trap density of states in organic field-effect transistors from experiment: A comparison of different methods, Phys. Rev. B, 81 (2010) 035327.

[18] Y.Y. Hu, Q. Qi, C. Jiang, Influence of different dielectrics on the first layer grain sizes and its effect on the mobility of pentacene-based thin-film transistors, Appl. Phys. Lett., 96 (2010) 133311.

[19] B. Bräuer, R. Kukreja, A. Virkar, H.B. Akkerman, A. Fognini, T. Tyliszczak, Z.N. Bao, Carrier mobility in pentacene as a function of grain size and orientation derived from scanning transmission X-ray microscopy, Org. Electron., 12 (2011) 1936-1942.

[20] S. Steudel, S. De Vusser, S. De Jonge, D. Janssen, S. Verlaak, J. Genoe, P. Heremans, Influence of the dielectric roughness on the performance of pentacene transistors, Appl. Phys. Lett., 85 (2004) 4400-4402.

[21] L.F. Deng, P.T. Lai, W.B. Chen, J.P. Xu, Y.R. Liu, H.W. Choi, C.M. Che, Effects of Different Annealing Gases on Pentacene OTFT With HfLaO Gate Dielectric, IEEE Electr Device L, 32 (2011) 93-95.

[22] R. Ruiz, B. Nickel, N. Koch, L.C. Feldman, R.F. Haglund, A. Kahn, G. Scoles, Pentacene ultrathin film formation on reduced and oxidized Si surfaces, Phys. Rev. B, 67 (2003) 125406.

[23] S.Y. Yang, K. Shin, C.E. Park, The effect of gate-dielectric surface energy on pentacene morphology and organic field-effect transistor characteristics, Adv. Funct. Mater., 15 (2005) 1806-1814.

[24] S. Verlaak, S. Steudel, P. Heremans, D. Janssen, M.S. Deleuze, Nucleation of organic semiconductors on inert substrates, Phys. Rev. B, 68 (2003) 195409.

[25] H. Yu, Y. Shen, Y. Cui, H. Qi, J. Shao, Z. Fan, Characterization of LaF3 coatings prepared at different temperatures and rates, Appl. Surf. Sci., 254 (2008) $1783-1788$.

[26] D.K. Owens, R.C. Wendt, Estimation of the surface free energy of polymers, J. Appl. Polym. Sci., 13 (1969) 1741-1747.

[27] B.J. Song, K. Hong, W.K. Kim, K. Kim, S. Kim, J.L. Lee, Effect of Oxygen Plasma Treatment on Crystal Growth Mode at Pentacene/Ni Interface in Organic Thin-Film Transistors, J. Phys. Chem. B, 114 (2010) 14854-14859.

[28] S.E. Fritz, T.W. Kelley, C.D. Frisbie, Effect of dielectric roughness on performance of pentacene TFTs and restoration of performance with a polymeric smoothing layer, J. Phys. Chem. B, 109 (2005) 10574-10577.

[29] J.T. Sadowski, Pentacene growth on 3-aminopropyltrimethoxysilane modified silicon dioxide, Opt. Mater., 34 (2012) 1653-1638.

[30] I.G. Hill, C.M. Weinert, L. Kreplak, B.P. van Zyl, Influence of self-assembled monolayer chain length on modified gate dielectric pentacene thin-film transistors, Appl. Phys. a-Mater., 95 (2009) 81-87. 


\section{Figure Caption}

Fig. 1 Output characteristics of the OTFTs with different plasma treatment times: (a) fresh; (b) after 6 months.

Fig. 2 Transfer characteristics of the OTFTs (with different plasma treatment times) at $\mathrm{V}_{\mathrm{D}}=-6 \mathrm{~V}$ : (a) fresh; (b) after 6 months.

Fig. 3 AFM image of the 3-nm pentacene film grown on $\mathrm{ZrLaO}$ dielectric: (a) without plasma treatment; (b) treated for $100 \mathrm{~s}$; (c) treated for $300 \mathrm{~s}$; (d) treated for $600 \mathrm{~s}$.

Fig. 4 XPS spectra of (a) La 3d and (b) $\mathrm{Zr} 3 \mathrm{~d}$ of $\mathrm{ZrLaO}$ with different fluorine-plasma treatment times.

Fig. 5 XPS spectrum of F 1s and leakage current density: (a) 100-s treatment; (b) 300-s treatment; (c) 600-s treatment; and (d) leakage current density versus gate voltage for the $\mathrm{Al} / \mathrm{ZrLaO} / \mathrm{Si}$ capacitor.

Fig. 6 Morphology of gate-dielectric surfaces with different plasma treatment times: (a) without treatment; (b) 100-s treatment; (c) 300-s treatment; (d) 600-s treatment. 
Fig. 1
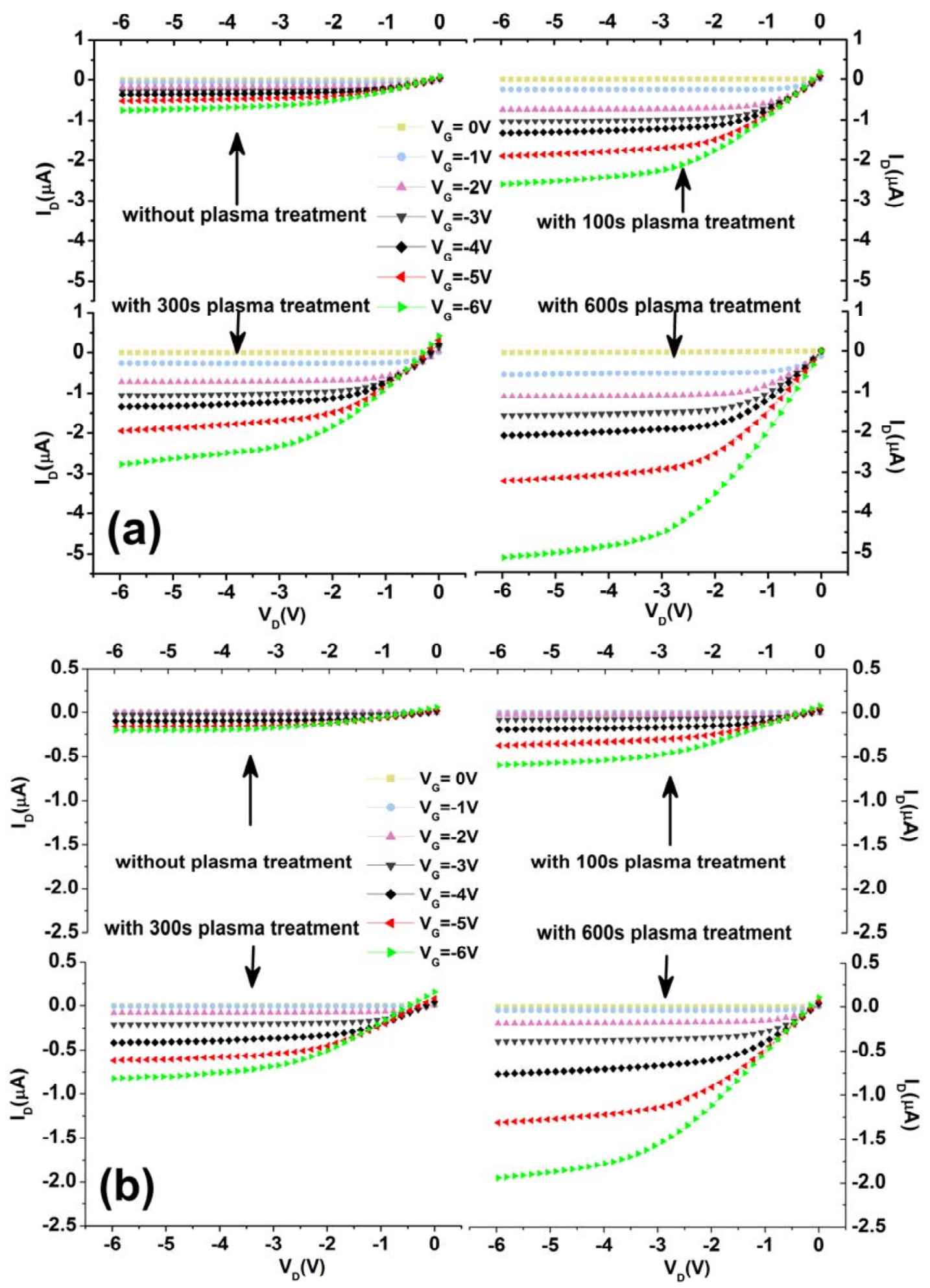
Fig. 2
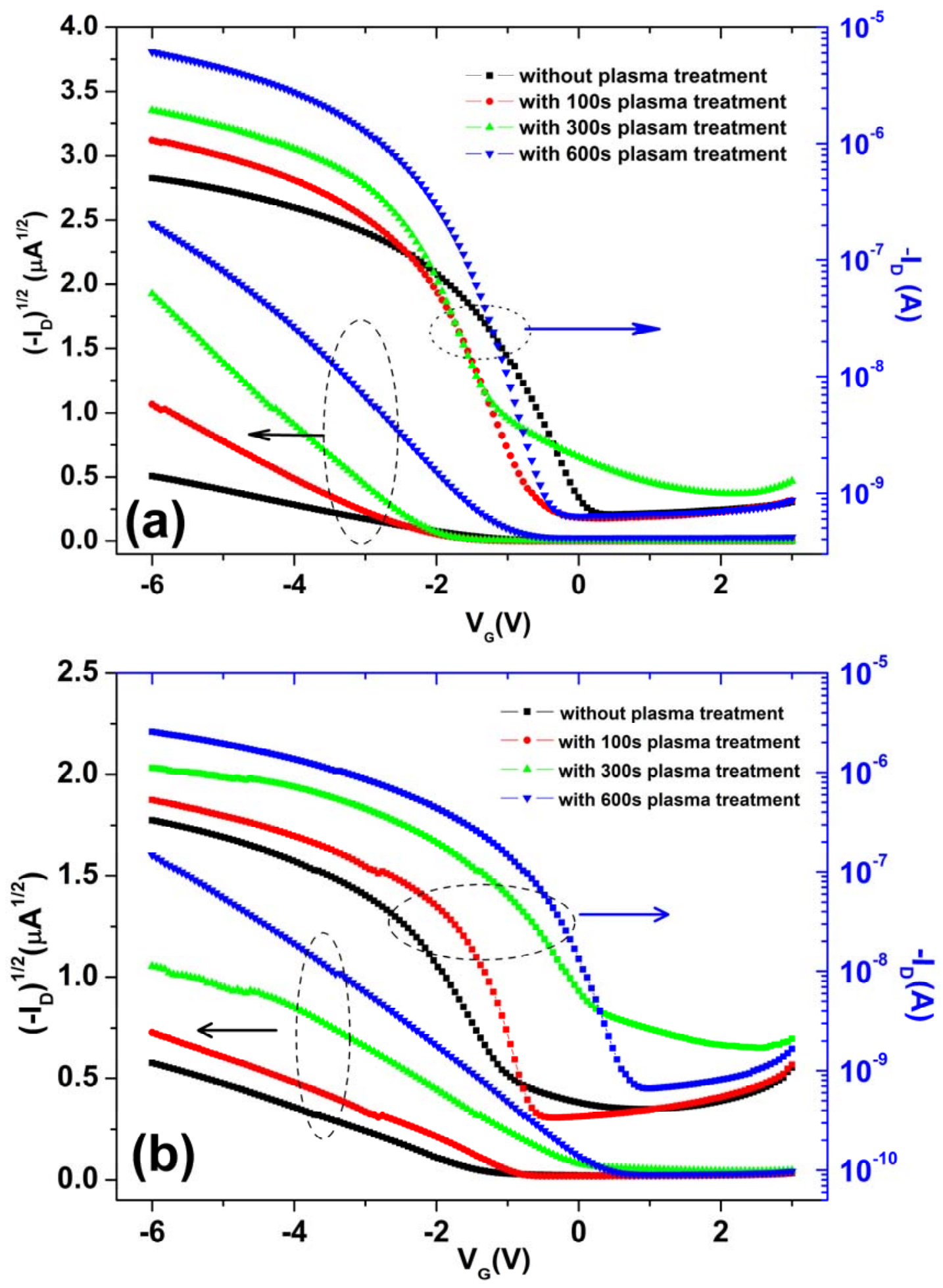
Fig. 3

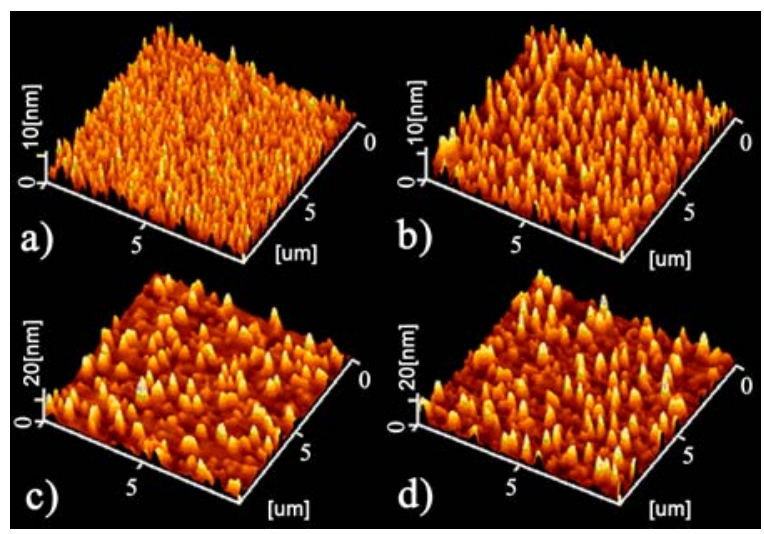


Fig. 4
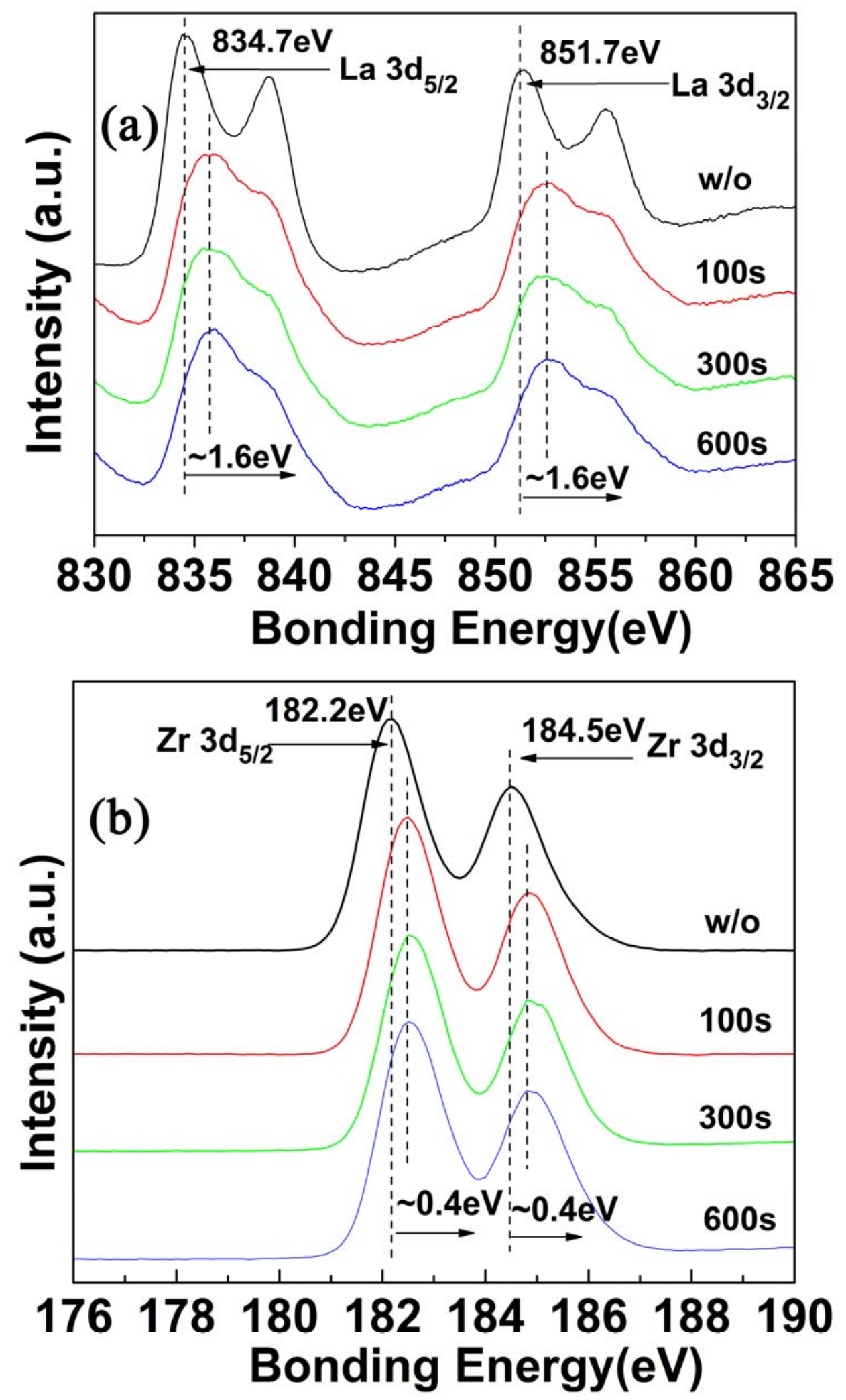
Fig. 5

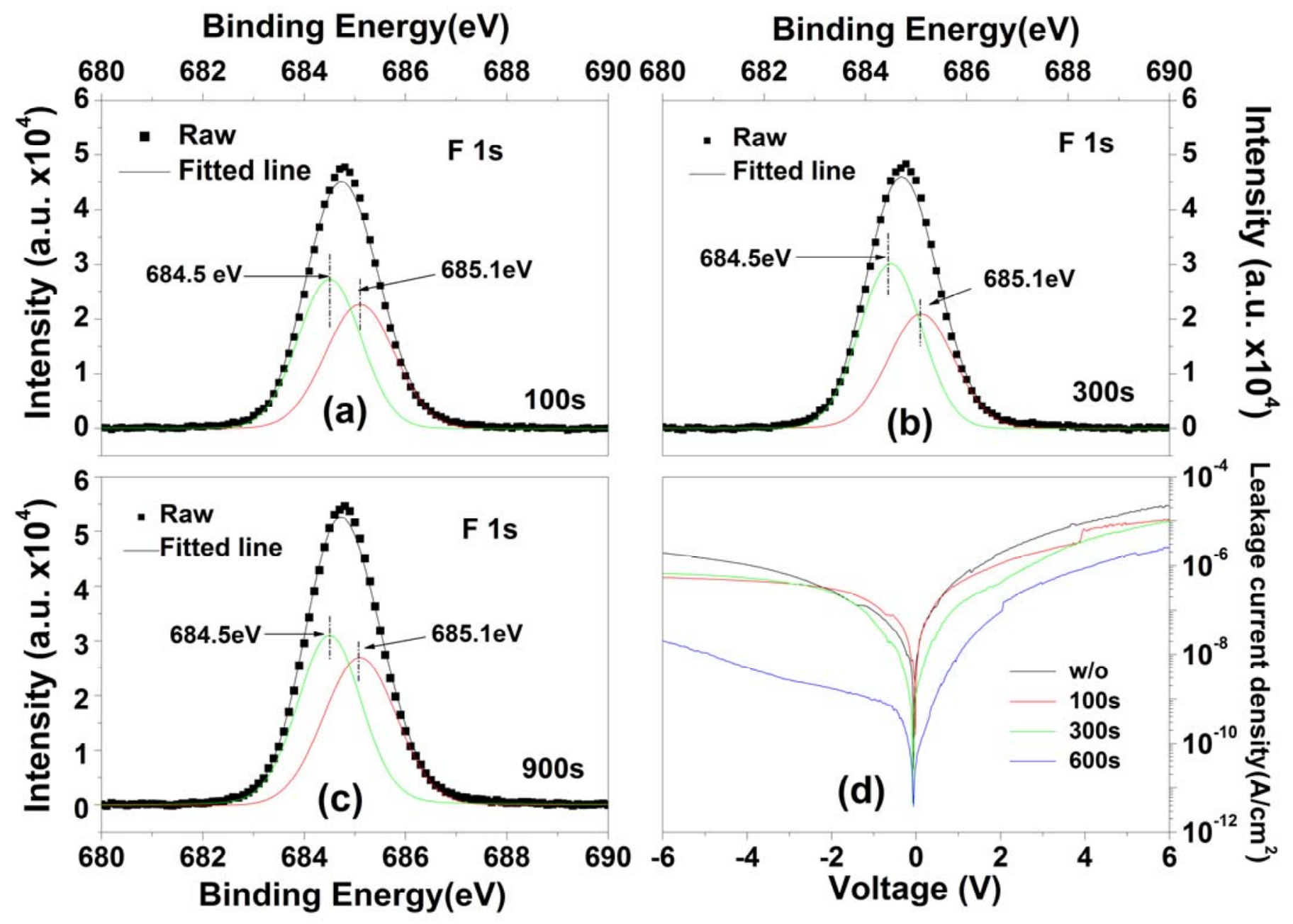


Fig. 6

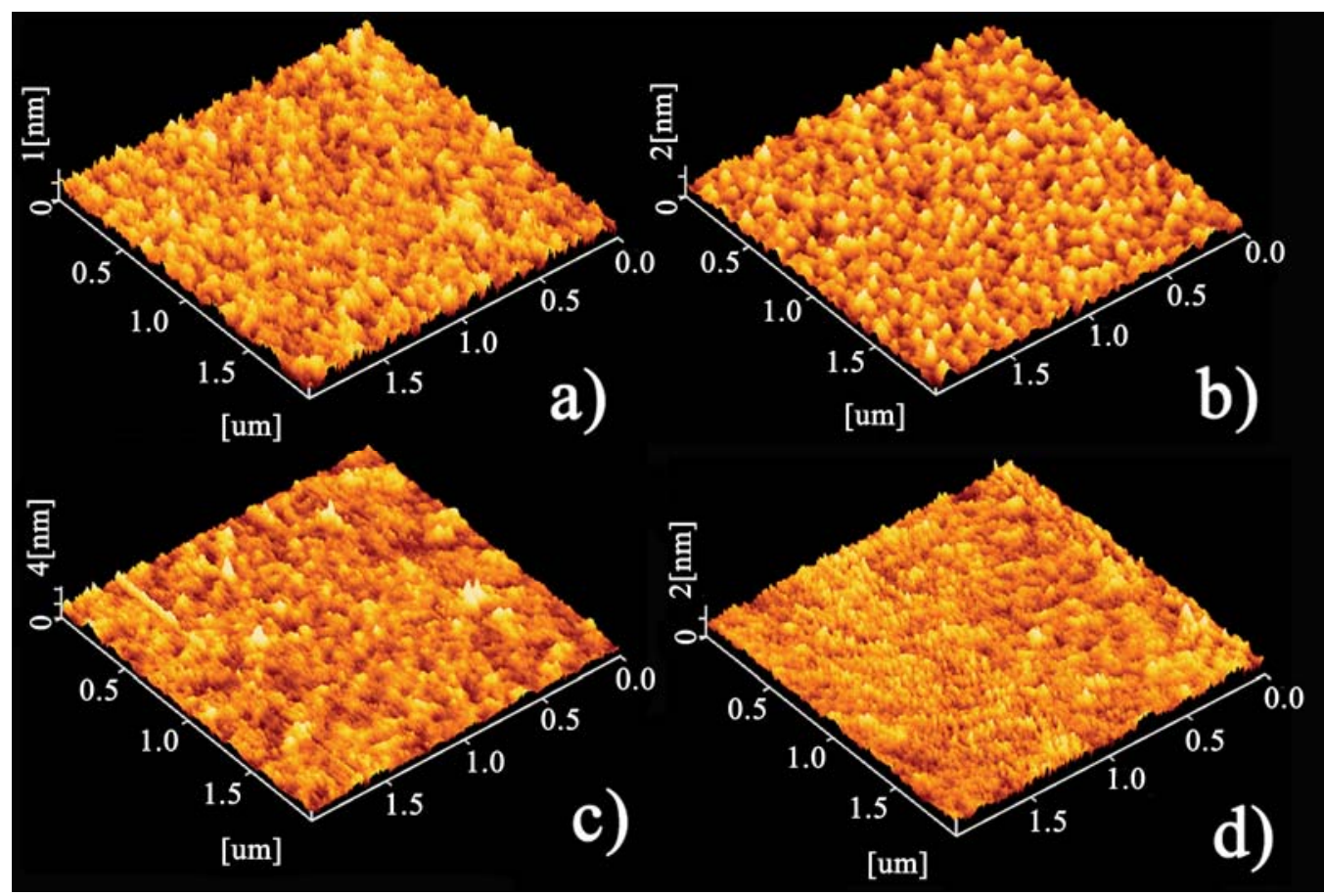


Table 1

Parameters of the OTFTs with different plasma treatment time (fresh)

\begin{tabular}{lcccc}
\hline \hline Plasma Treatment Time & $0 \mathrm{~s}$ & $100 \mathrm{~s}$ & $300 \mathrm{~s}$ & $600 \mathrm{~s}$ \\
\hline Carrier Mobility $\left(\mathrm{cm}^{2} / \mathrm{V} \cdot \mathrm{s}\right)$ & 0.031 & 0.112 & 0.443 & 0.717 \\
\hline Threshold Voltage $(\mathrm{V})$ & -0.723 & -1.04 & -1.34 & -0.971 \\
\hline $\mathrm{SS}(\mathrm{V} / \mathrm{dec})$ & 0.796 & 0.715 & 0.659 & 0.578 \\
\hline $\mathrm{On} / \mathrm{Off}$ Current Ratio $\left(10^{3}\right)$ & 1.09 & 1.75 & 1.93 & 9.65 \\
\hline $\mathrm{I}_{\mathrm{D}}\left(\mathrm{V}_{\mathrm{DS}}=\mathrm{V}_{\mathrm{GS}}=-6 \mathrm{~V}\right)(\mu \mathrm{A})$ & 0.554 & 0.939 & 1.93 & 6.11 \\
\hline $\mathrm{Cox}\left(\mu \mathrm{F} / \mathrm{cm}^{2}\right)$ & 0.244 & 0.233 & 0.235 & 0.236 \\
\hline $\mathrm{t}_{\mathrm{ox}}(\mathrm{nm})$ & 40.1 & 40.1 & 40.0 & 40.0 \\
\hline$\kappa$ & 11.0 & 10.5 & 10.6 & 10.6 \\
\hline $\begin{array}{l}\text { Dielectric Surface } \\
\text { Roughness }(\mathrm{nm})\end{array}$ & 0.19 & 0.30 & 0.30 & 0.29 \\
\hline Trap Density $\left(10^{13} \mathrm{~cm}^{-2} \mathrm{eV}^{-1}\right)$ & 1.88 & 1.60 & 1.48 & 1.28 \\
\hline \hline
\end{tabular}


Table 2

Parameters of the OTFTs with different plasma treatment time (after 6 months)

\begin{tabular}{lcccc}
\hline \hline Plasma Treatment Time & $0 \mathrm{~s}$ & $100 \mathrm{~s}$ & $300 \mathrm{~s}$ & $600 \mathrm{~s}$ \\
\hline Carrier Mobility $\left(\mathrm{cm}^{2} / \mathrm{V} \cdot \mathrm{s}\right)$ & 0.01 & 0.048 & 0.076 & 0.102 \\
\hline Threshold Voltage $(\mathrm{V})$ & -1.238 & -0.765 & -0.419 & -0.356 \\
\hline $\mathrm{SS}(\mathrm{V} /$ dec $)$ & 0.81 & 0.68 & 0.71 & 0.63 \\
\hline On/Off Current Ratio $\left(10^{3}\right)$ & 0.81 & 1.56 & 0.85 & 3.86 \\
\hline $\mathrm{I}_{\mathrm{D}}\left(\mathrm{V}_{\mathrm{DS}}=\mathrm{V}_{\mathrm{GS}}=-6 \mathrm{~V}\right)(\mu \mathrm{A})$ & 0.33 & 0.59 & 1.11 & 2.56 \\
\hline Trap Density $\left(10^{13} \mathrm{~cm}^{-2} \mathrm{eV}^{-1}\right)$ & 1.92 & 1.52 & 1.60 & 1.41 \\
\hline \hline
\end{tabular}


Table 3

Characteristics of 3-nm pentacene films grown on $\mathrm{ZrLaO}$ dielectrics with different plasma treatment time

\begin{tabular}{lcccc}
\hline \hline Plasma Treatment Time & $0 \mathrm{~s}$ & $100 \mathrm{~s}$ & $300 \mathrm{~s}$ & $600 \mathrm{~s}$ \\
\hline Mean Size $\left(\mathrm{nm}^{2}\right) \times 10^{5}$ & 0.629 & 0.775 & 1.16 & 1.01 \\
\hline Mean Diameter $(\mathrm{nm}) \times 10^{2}$ & 2.83 & 3.14 & 3.84 & 3.58 \\
\hline Count $(10 \mu \mathrm{m} \times 10 \mu \mathrm{m})$ & 554 & 271 & 145 & 157 \\
\hline Grain Area $\left(\mu \mathrm{m}^{2}\right)$ & 348 & 210 & 168 & 158 \\
\hline Ratio of Grain Area $(\%)$ & 34.9 & 21.1 & 16.8 & 15.9 \\
\hline RMS roughness $(\mathrm{nm})$ & 2.69 & 3.13 & 3.79 & 3.65 \\
\hline \hline
\end{tabular}


Table 4

Surface energy of $\mathrm{ZrLaO}$ film with different plasma treatment time and the fluorine atomic concentration at the surface of the film

\begin{tabular}{lcccc}
\hline \hline Plasma Treatment Time & $0 \mathrm{~s}$ & $100 \mathrm{~s}$ & $300 \mathrm{~s}$ & $600 \mathrm{~s}$ \\
\hline Dispersion $\left(\mathrm{mJm}^{-2}\right)$ & 29.0 & 41.7 & 41.3 & 41.3 \\
\hline Polar $\left(\mathrm{mJm}^{-2}\right)$ & 14.2 & 16.1 & 20.0 & 20.9 \\
\hline Surface energy $\left(\mathrm{mJm}^{-2}\right)$ & 43.2 & 57.8 & 61.3 & 62.2 \\
\hline F atomic concentration $(\%)$ & - & 16.9 & 17.1 & 20.3 \\
\hline \hline
\end{tabular}

